\title{
Demographic Trends in Carcinoma Esophagus from India along with a Brief Comparative Review of the Global Trends
}

\author{
Arvind Krishnamurthy ${ }^{1}$ Siva Shankar Behuria ${ }^{1}$ \\ ${ }^{1}$ Departments of Surgical Oncology, Cancer Institute (WIA), \\ Chennai, Tamil Nadu, India
}

\section{South Asian J Cancer 2020;9:163-167.}

\begin{abstract}
Address for correspondence Dr. Arvind Krishnamurthy, MS (Gen Surg) MCh ( Surgical Oncology), DNB (Surgical Oncology), Departments of Surgical Oncology, Cancer Institute (WIA), 38, Sardar Patel Rd, Chennai 600036, Tamil Nadu, India (e-mail: drarvindkrishnamurthy@yahoo.co.in).
\end{abstract}

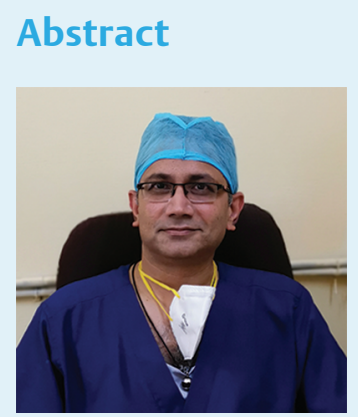

Dr. Arvind Krishnamurthy, MS ( Gen Surg) MCh

(Surgical Oncology), DNB (Surgical Oncology)

Keywords

- adenocarcinoma

- demographics

- epidemiology

- esophageal cancer

- India

- risk factors

- squamous cell carcinoma
Background Esophageal cancers (ECs) are more prevalent in the East Asian countries of the world, wherein squamous cell carcinomas (SCCs) are the predominant histological subtype. In contrast, the patterns in the West are a bit heterogeneous, with esophageal adenocarcinoma (AC) being the more frequent histological subtype. There is very sparse published Indian data pertaining to the demographic trends of ECs.

Materials and Methods Our study was a retrospective analysis of the demographic trends of 917 patients afflicted with ECs who were managed at our center over a 10 -year period.

Results and Discussion EC accounted for nearly $4.1 \%$ of the total cancer burden managed at our center from January 2002 to December 2011. The mean age of our patient cohort was 54.2 years. The male:female ratio was nearly 1.7:1. Tobacco chewing was noted in $25.4 \%$, smoking in $37 \%$, while alcohol consumption was noted in approximately $20 \%$ of the patients. SCC was the most common histological subtype (78.3\%), while ACs constituted only 9.9\%. Eighty-nine percent of our patients presented with locally advanced staged tumors. Definitive chemoradiation was the most common modality of definitive management then; however, over the years, our preferred choice of the management of ECs has shifted to neoadjuvant chemoradiation, followed by surgery in the carefully selected patients of locally advanced resectable ECs.

Conclusion Our study clearly shows SCC to be the most common histological subtype among ECs, a trend that has been observed in the vast majority of the East Asian nations. The epidemic rise in the incidence of esophageal ACs as seen in the West is not seen in our study. Periodic monitoring of the demographic trends of ECs is of great importance both for clinicians and policymakers. We hope that our study will enlighten both policy holders and clinicians to better channelize the efforts toward prevention and more effective management of this deadly cancer.
How to cite this article: Krishnamurthy A, Behuria SS. Demographic Trends in Carcinoma Esophagus from India along with a Brief Comparative Review of the Global Trends. South Asian J Cancer 2020;9(3):163-167.

DOI https://doi.org/10.1055/s-0041-1726139 ISSN 2278-330X. (c) 2020. MedIntel Services Pvt Ltd.

This is an open access article published by Thieme under the terms of the Creative Commons Attribution-NonDerivative-NonCommercial-License, permitting copying and reproduction so long as the original work is given appropriate credit. Contents may not be used for commercial purposes, or adapted, remixed, transformed or built upon. (https://creativecommons.org/licenses/by-nc-nd/4.0/).

Thieme Medical and Scientific Publishers Private Ltd A-12, Second

Floor, Sector -2, NOIDA -201301, India 


\section{Introduction}

Carcinoma of the esophagus ranks seventh in terms of incidence $(572,000$ new cases) and sixth in terms of the overall mortality worldwide (509,000 deaths). Esophageal cancer (EC) was, in fact, responsible for an estimated 1 in every 20 cancer deaths globally in the year 2018. ${ }^{1}$ Squamous cell carcinoma (SCC) conventionally was the most common histological variant worldwide; however, over the past few decades, the number of patients diagnosed with esophageal and gastroesophageal junction adenocarcinomas (ACs) has markedly increased, and this increase has been primarily attributed to the increasing rates of obesity and gastroesophageal reflux disease. ${ }^{2}$ However, in less developed and developing nations, including parts of China, India, and Central Asia, SCC continues to remain the most common histological variant. ${ }^{3-6}$ We analyzed the demographic trends of ECS that presented to our tertiary care center over a 10 -year period.

\section{Materials and Methods}

Our study is a retrospective analysis of the medical records of patients managed at our tertiary care center between January 2002 and December 2011. Nine hundred and seventeen $(n=917)$ EC patients registered in our hospital in the said period. The demographic data pertaining to age, sex, body mass index (BMI), habits (chewing tobacco, smoking either bidi or cigarette, and alcohol consumption), dietary habits, and presenting symptoms were captured from the case records and further analyzed. The tumor in the esophagus was further analyzed, based on location, histology, grade, endoscopic findings, stage, and the intent of treatment.

\section{Results}

\section{Age and Sex}

Nine hundred and seventeen patients in our study included 572 males (62\%) and 345 females (38\%), with a male-to-female ratio of $1.7: 1$. The mean age of our patient cohort was 54.2 years (age 18-88 years).

\section{Tobacco, Alcohol, and Eating Habits}

All forms of tobacco consumption were noted in our patient cohort. Two hundred and thirty-three (25\%) patients were tobacco chewers, which included 157 (67\%) males and 76 (31\%) females $(p=0.05)$. Our cohort also included 337 patients with a history of tobacco smoking (either cigarettes or bidi); the vast majority were men 328 (97\%). Furthermore, 176 (20\%) patients were reported to consume alcohol; the vast majority of them were again males $(n=173$ [98\%]), which was significantly higher than what was noted among the females $(p<0.05)$.

The mean duration (in years) of exposure to chewing tobacco, smoking, and consumption of alcohol was 28.7 ( $2-60$ years), 24.6 ( $0.5-55$ years), and 24.6 ( $1-50$ years) years, respectively. Nonvegetarian diet was noted to be the most common dietary pattern in nearly $776(85 \%)$ patients. This group consisted of 486 (63\%) males and 135 (15\%) females; this association was found to be not statistically significant $(p=0.312)$.

\section{Clinical Presentation}

Dysphagia was the most common presenting symptom seen in $96 \%$ of our patients. The other common clinical presentations included vomiting and weight loss, and the less common presenting symptoms included odynophagia, pain abdomen, hoarseness of voice, cough, and neck node enlargement.

\section{Body Mass Index}

The mean Body Mass Index (BMI) in our patient cohort was 19.72 (range: 12-49). The mean BMI among the male patients was 19.81 (12-49), while it was 19.57 (13-31) among the female patients $(p=0.61)$.

\section{Location}

The incidence of lower-third ECs (32-40 cm) was 388 (42.3\%), which was slightly higher than middle-third ECs $(24-32 \mathrm{~cm})$ 377 (41\%).

\section{Histology}

SCC was the most common histological subtype in our cohort ( $n=718$ [78\%]), while 91 patients (9.9\%) had ACs. SCC, in fact, was the most common histology across all locations of the esophagus (325 patients in the middle-third esophagus and 269 patients in the lower-third esophagus). The vast majority of the ACs $(n=85)$ were found in the lower-third of the esophagus $(p=0.0000)$. Further, the vast majority of the ECs in our cohort were grade 3 .

The mean age of presentation in $\mathrm{AC}$ was significantly higher than what was observed in SCC $(p=0.001)$. The male:female ratio of SCC was 1.44:1 (424:294), whereas it was 5:1 (76:15) for ACs, which was also found to be statistically significant $(p=0.00006)$. Further, we found that the incidence of SCC among the age group $40-60$ years and $>60$ years was significantly higher to the $<40$ years age group as compared with the AC group $(p=0.024)$.

Interestingly, our study did not find any statistical significance between SCC and ACs in relation to the habits of chewing tobacco, smoking, alcohol, dietary pattern, BMI, morphological type of lesion, and the intent of treatment.

One hundred and sixty-seven patients of 718 SCC and 32 patients of $91 \mathrm{AC}$ patients underwent upfront surgery either a transthoracic or transhiatal surgery. Two hundred and thirty-five patients in SCC group received definitive chemoradiation, whereas two patients in AC group received definitive chemoradiation.

\section{Stage of Presentation and Management}

The vast majority of our patients ( $n=821$ [89\%]) clinically presented with locally advanced stages, and only $3 \%$ (27 patients) presented with early-stage disease. A potentially curative treatment was offered to 597 (65\%) patients, of which 268 (29\%) patients received definitive chemoradiotherapy, 220 (24\%) received upfront surgery, and 70 patients (8\%) received definitive radiotherapy. Two hundred and sixty-four patients 
(29\%) received palliative treatment. Furthermore, 69 patients (8\%) were found to harbor metastatic disease at presentation, more commonly seen in the liver, followed by the lung.

\section{Discussion}

A great deal of heterogeneity is noted in terms of epidemiology, molecular biology, clinical behavior, and management of ECs worldwide.

\section{Global Burden}

The global incidence of ECs shows a wide variation, being highest in the Asian belt between Iran, Turkey, and north-central China. ${ }^{78}$ The EC incidence was much lesser in the Western countries; however, over the past several decades, the number of patients diagnosed with $A C$ is increasing, probably due to the high prevalence of obesity, Barrett's esophagus, and chronic gastroesophageal reflux disease. ${ }^{1,2}$ This trend is further reflected by the fact that the age standardized rate (ASR) for carcinoma esophagus in 2018 was reported to be the highest in East Asia. The ASR stood at 19.9/100,000 for men, while it was $6.8 / 100,000$ for women. ${ }^{1}$ The ASRs were reported to be the lowest in West Africa, where the rates among men are $1.6 / 100,000$, while the similar rates among women stand at $0.8 / 100,000 .{ }^{1}$

It has been reported that in countries with higher human development index (HDI), there is a higher incidence of AC of the esophagus as evidenced in the US, where the incidence of AC of the esophagus has increased by over $400 \%$ over the past 25 years. ${ }^{9,10}$ Among the Asian countries, China and Singapore have also reported an increasing incidence of esophageal $A C{ }^{11,12}$ The corresponding decline in the incidence of esophageal SCC in the above nations is believed to have been preceded by economic gains and dietary improvements, while in several high-income countries, it is believed that these reductions were primarily caused by a decline in the incidence of cigarette smoking. In contrast, in countries with lower HDI like India, there is a higher incidence of esophageal SCC.

\section{Indian Burden}

EC occupies the sixth position both in terms of incidence (4.9\% of all cancers) and mortality (5.9\% of all cancers) in India for the year 2018. The absolute number of EC cases for the year 2018 are 52,396 and the number of deaths being 46,504. ${ }^{1}$ Among men, ECs does figure in the list of top 5 cancers (number 5); the number of new cases in 2018 among men was 33,890. However, EC does not find a mention among the top 5 cancers among women. ${ }^{1}$ In some parts of India, the incidence of EC was very high, for example, in Jammu and Kashmir and nearly all the states from the north-eastern parts of India. ${ }^{12}$

\section{Comparative Demographic Trends}

Population-based data worldwide suggest that ECs peak in the sixth and seventh decades of life.13,14 The mean age of presentation in our cohort was 54.2 years, which is, in fact, a decade earlier than the clinical presentations from the rest of the world. Approximately $70 \%$ of ECs globally occurred in men, and there is reported to be a 2 to 3 -fold difference in incidence and mortality rates between the sexes worldwide. Our study too showed a male preponderance with a male-to-female incidence ratio of 1.7:1.

Our study clearly showed SCC to be the most common histological subtype, 718 (78\%), followed by AC 91 (9.9\%). This finding is in line with the findings that SCC remains the most common histologic subtype in many Asian populations and most of them located in the middle- or lower-third of the esophagus. ${ }^{15,16}$ Our study further reiterated the finding that the mean age of presentation of AC was significantly higher than the age of presentation for SCC.

A plethora of factors contribute to the causation of SCC, and there are in fact distinct variations between the populations of the East and West. Tobacco and alcohol have been associated with $\mathrm{SCC}^{8,17}$ while obesity, gastroesophageal reflux, and Barrett's esophagus have been linked to AC. ${ }^{18,19}$ However, this classical association was not in our patient cohort. In a population-based US study, among patients with SCC, the factors associated with a higher population-attributable risks for SCC included tobacco smoking, alcohol use, and inadequate consumption of fruits and vegetables..$^{20}$ However, a prospective study from China showed that smoking has only a modest impact on the development of SCC, and drinking alcohol was not associated with an increased risk. ${ }^{21} \mathrm{~A}$ meta-analysis study by Kubo and Corley ${ }^{22}$ suggests high BMI (> 25) was associated with an increased risk of esophageal AC.

The other risk factors implicated in the causation of ECs include consumption of very hot liquids, lack of fruits and vegetables, consumption of mate tea, poor oral hygiene, opioid use, polycyclic aromatic hydrocarbon exposure, chewing betel nuts, and association with Helicobacter pylori infection among others. ${ }^{23-25}$

As per the Japanese Society for Esophageal Disease Guidelines, six different types (types 0-5) of esophageal tumor/lesion are found during endoscopy, which is based on macroscopic appearance. ${ }^{26}$ The common morphological findings on endoscopy in our series were ulceroproliferative (combined type), circumferential ulcerative (type $2 / 3$ ), and the proliferative type (type 1 ) in decreasing order.

The management of ECs has witnessed a major change over the years among a remarkable change in histology and epidemiology. ${ }^{27}$ Historically, radical surgery alone was considered to be the sole curative treatment for ECs. ${ }^{28,29}$ In the present day, surgery alone in the form of either esophagectomy alone or endoscopic resections has been reserved for in situ or early-stage disease. However, such tumors constituted a miniscule $3 \%$ of our entire cohort. The vast majority of our patients (around $89 \%$ ) were locally advanced. Definitive chemoradiation was an acceptable modality of treatment for locally advanced ECs in the previous decade, and hence, majority of our locally advanced ECs were managed by this modality. Definitive chemoradiation in the present day is only offered to patients with unresectable disease, patients who either are poor surgical candidates, or those who refuse surgery. ${ }^{27}$ It is noteworthy to state that a fraction of patients (7.6\%) in our cohort were 
deemed unfit for definitive chemoradiation and only had radiation as a treatment modality.

The management of locally advanced carcinomas of the esophagus has undergone a major evolution, with the widespread use of combined modality therapy based on high-level evidence from several randomized controlled trials (RCTs) and meta-analyses; neoadjuvant treatment is the new standard of care for locally advanced resectable ECs. ${ }^{30}$ However, a major unanswered question remains as to whether neoadjuvant chemoradiation is clearly superior to neoadjuvant chemotherapy. Following the publication of the results of CROSS trial, ${ }^{31}$ our preferred choice of the management of locally advanced ECs has shifted to neoadjuvant chemoradiation followed by surgery. ${ }^{30}$

There is very sparse published Indian data pertaining to the demographic trends of ECs, the most of which are case-control studies. ${ }^{12,32,33}$ The main limitation of this study was that it was based on retrospective data gathered from a single tertiary care center. The strengths of our study are the large number over a decade, and its reliability of the data acquisition, as the author's institution has cancer registries that capture both the hospital-based and the population-based data on various cancers. Our study, thus, adds to the limited published Indian data on the demography of ECs.

\section{Conclusion}

Our study clearly shows SCC to be the most common histological subtype among ECs, a trend that has been observed in the vast majority of East Asian nations. The epidemic rise in the incidence of esophageal ACs, as seen in the West and certain parts of Asia with a high HDI, is not seen in our study. ACs, in fact, constituted $<10 \%$ of our cases in our series. Periodic monitoring of the demographic trends of ECs is of great importance both for clinicians and policymakers. We hope that our study will enlighten both policyholders and clinicians to better channelize the efforts toward prevention and more effective management of this deadly cancer.

Funding
Nil.
Conflict of Interest
None declared.

\section{References}

1 Bray F, Ferlay J, Soerjomataram I, Siegel RL, Torre LA, Jemal A. Global cancer statistics 2018: GLOBOCAN estimates of incidence and mortality worldwide for 36 cancers in 185 countries. CA Cancer J Clin 2018;68(6):394-424

2 Blot WJ, Tarone RE, Esophageal cancer. In: Thun MJ, Linet MS, Cerhan JR, Haiman CA, Schottenfeld D, eds. Cancer Epidemiology and Prevention. 4th ed. New York: Oxford University Press; 2018:579-92

3 Arnold M, Laversanne M, Brown LM, Devesa SS, Bray F. Predicting the future burden of esophageal cancer by histological subtype: International trends in incidence up to 2030. Am J Gastroenterol 2017;112(8):1247-1255
4 Kamangar F, Dores GM, Anderson WF. Patterns of cancer incidence, mortality, and prevalence across five continents: defining priorities to reduce cancer disparities in different geographic regions of the world. J Clin Oncol 2006;24(14):2137-2150

5 Murphy G, McCormack V, Abedi-Ardekani B, et al. International cancer seminars: a focus on esophageal squamous cell carcinoma. Ann Oncol 2017;28(9):2086-2093

6 Pennathur A, Gibson MK, Jobe BA, Luketich JD. Oesophageal carcinoma. Lancet 2013;381(9864) :400-412

7 Eslick GD. Epidemiology of esophageal cancer. Gastroenterol Clin North Am 2009;38(1):17-25, vii vii.

8 Bray F, Jemal A, Grey N, Ferlay J, Forman D. Global cancer transitions according to the Human Development Index (2008-2030): a population-based study. Lancet Oncol 2012;13(8):790-801

9 Brown LM, Devesa SS, Chow WH. Incidence of adenocarcinoma of the esophagus among white Americans by sex, stage, and age. J Natl Cancer Inst 2008;100(16):1184-1187

10 ZhangH,ChenSH,LiYM.Epidemiologicalinvestigationofesophageal carcinoma. World J Gastroenterol 2004;10(12):1834-1835

11 Fernandes ML, Seow A, Chan YH, Ho KY. Opposing trends in incidence of esophageal squamous cell carcinoma and adenocarcinoma in a multi-ethnic Asian country. Am J Gastroenterol 2006;101(7):1430-1436

12 Dar NA, Shah IA, Bhat GA, et al. Socioeconomic status and esophageal squamous cell carcinoma risk in Kashmir, India. Cancer Sci 2013;104(9):1231-1236

13 Enzinger PC, Mayer RJ. Esophageal cancer. N Engl J Med 2003;349(23):2241-2252

14 Malkan G, Mohandas KM. Epidemiology of digestive cancers in India. I. General principles and esophageal cancer. Indian J Gastroenterol 1997;16(3):98-102

15 Mustafa SA, Banday SZ, Bhat MA, Patigaroo AR, Mir AW, Bhau KS. Clinico-epidemiological profile of esophageal cancer in Kashmir. Int J Sci Stud 2016;3:197-202

16 Zhang HZ, Jin GF, Shen HB. Epidemiologic differences in esophageal cancer between Asian and Western populations. Chin J Cancer 2012;31(6):281-286

17 Esophageal cancer: epidemiology, pathogenesis and prevention. Nat Clin Pract Gastroenterol Hepatol 2008;5(9):517-526

18 Coleman HG, Xie SH, Lagergren J. The epidemiology of esophageal adenocarcinoma. Gastroenterology 2018;154(2):390-405

19 Spechler SJ. Barrett esophagus and risk of esophageal cancer: a clinical review. JAMA 2013;310(6):627-636

20 Engel LS, Chow WH, Vaughan TL, et al. Population attributable risks of esophageal and gastric cancers. J Natl Cancer Inst 2003;95(18):1404-1413

21 Tran GD, Sun XD, Abnet CC, et al. Prospective study of risk factors for esophageal and gastric cancers in the Linxian general population trial cohort in China. Int J Cancer 2005;113(3):456-463

22 Kubo A, Corley DA. Body mass index and adenocarcinomas of the esophagus or gastric cardia: a systematic review and meta-analysis. Cancer Epidemiol Biomarkers Prev 2006;15(5):872-878

23 Lubin JH, De Stefani E, Abnet CC, et al. Maté drinking and esophageal squamous cell carcinoma in South America: pooled results from two large multicenter case-control studies. Cancer Epidemiol Biomarkers Prev 2014;23(1):107-116

24 Islami F, Pourshams A, Nasrollahzadeh D, et al. Tea drinking habits and oesophageal cancer in a high risk area in northern Iran: population based case-control study. BMJ 2009;338:b929

25 Loomis D, Guyton KZ, Grosse Y, et al; International Agency for Research on Cancer Monograph Working Group. Carcinogenicity of drinking coffee, mate, and very hot beverages. Lancet Oncol 2016;17(7):877-878

26 Japanese Society for Esophageal Disease, Guidelines for the Clinical and Pathologic Studies on Carcinoma of the Esophagus. 11th ed. Tokyo: Kanehara Co.; 2015 
27 Moaven O, Wang TN. Combined modality therapy for management of esophageal cancer: Current approach based on experiences from East and West. Surg Clin North Am 2019;99(3):479-499

28 Ganesamoni S, Krishnamurthy A. Three-field transthoracic versus transhiatal esophagectomy in the management of carcinoma esophagus-a single-center experience with a review of literature. J Gastrointest Cancer 2014;45(1):66-73

29 Ravishankaran P, Krishnamurthy A. Prognostic value of metastatic lymph nodal ratio in squamous cell carcinoma of esophagus: A three-step extrapolative study. South Asian J Cancer 2014;3(4):213-216

30 Krishnamurthy A, Mohanraj N, Radhakrishnan V, John A, Selvaluxmy G. Neoadjuvant chemoradiation for locally advanced resectable carcinoma of the esophagus:
A single-center experience from India with a brief review of the literature. Indian J Cancer 2017;54(4):646-651

31 Shapiro J, van Lanschot JJ, Hulshof MCC, et al; CROSS study group. Neoadjuvant chemoradiotherapy plus surgery versus surgery alone for oesophageal or junctional cancer (CROSS): long-term results of a randomised controlled trial. Lancet Oncol 2015;16(9):1090-1098

32 Chitra S, Ashok L, Anand L, Srinivasan V, Jayanthi V. Risk factors for esophageal cancer in Coimbatore, southern India: a hospital-based case-control study. Indian J Gastroenterol 2004;23(1):19-21

33 Nandakumar A, Anantha N, Pattabhiraman V, et al. Importance of anatomical subsite in correlating risk factors in cancer of the oesophagus - report of a case-control study. Br J Cancer 1996;73(10):1306-1311 